\title{
A model of the response of GMR of metallic multilayers to external magnetic field
}

\author{
J.I. UBA ${ }^{1 *}$, A.J. ЕкPUNOBI ${ }^{2}$, P.I. EKWO ${ }^{2}$ \\ ${ }^{1}$ Department of Physics, Nwafor Orizu College of Education, P.M.B. 1734, Onitsha, Anambra, Nigeria \\ ${ }^{2}$ Department of Physics/Industrial Physics, Nnamdi Azikiwe University, P.M.B. 5025, Awka, Anambra, Nigeria
}

\begin{abstract}
It has not been possible to transform resistivity models in terms of magnetic field in order to account for variation of giant magnetoresistance (GMR) with external magnetic field, which would have led to determination of material properties. This problem is approached mathematically via variation calculus to arrive at an exponential function that fits observed GMR values. Using this model in free electron approximation, the mean Fermi vector, susceptibility and total density of states of a number of metallic multilayers are determined from their reported GMR values. Susceptibility is found to depend on interface roughness and antiferromagnetic (AF) coupling; thus, it gives qualitative measure of interface quality and AF coupling. Comparison of susceptibilities and GMRs of electrodeposited and ion beam sputtered $\mathrm{Co} / \mathrm{Cu}$ structures shows that a rough interface suppresses GMR in the former but enhances it in the latter.
\end{abstract}

Keywords: giant magnetoresistance (GMR); metallic multilayer; susceptibility

(C) Wroclaw University of Technology.

\section{Introduction}

Perpendicular and in-plane electronic transport in metallic multilayers (MML) have been modelled in classical [1-3] and quantum [4-6] formalisms. Though these resistivity models give good account of the giant magnetoresistance (GMR), especially as functions of thickness, interface roughness and mean free path $[3,6]$, experimental result is always a function of magnetic field $\mathrm{B}$ and none of the resistivity models can be expressed in terms of $\mathrm{B}$ to account for this variation. The reason is that the knowledge of $\mathrm{B}$ dependence on angle $\Theta$ between magnetizations of the ferromagnetic layers is required [3]. $\Theta$ itself, is determined by Zeeman anisotropy and exchange energies, which are dependent on the properties of involved system and prevailing conditions. For this reason, in models, where $\Theta$ features explicitly, arbitrary values are assigned to it [3].

Experiments show that GMR reduces with increasing magnetization up to a saturation field,

*E-mail: ikemuba@gmail.com where further magnetization is not possible. Going by the behavior of metals in magnetic field, such change is associated with redistribution of spin up and spin down electrons. The redistribution is proportional to magnetization, with Pauli susceptibility as a proportionality constant. Therefore, at the saturation field this spin redistribution ceases or rather attains a constant value since further magnetization is not possible and the GMR attains its asymptotic (maximum) value. If there was a model that showed a response of GMR magnitude to $B$, then both quantitative measure of spin redistribution and susceptibility of the involved multilayer could be deduced. Such model would be a characterization tool.

If any of the established resistivity models included magnetic field explicitly, it would have been easy to describe GMR variation with B. However, since each of the resistivity models gives quantitative values that are implemented in the magnetoresistance equation, it is possible to use the latter to get the response of GMR to magnetic field. In this paper, such model is presented and used to deduce the size of spin redistribution, susceptibility and 
effective Fermi vector of some multilayers from their reported giant magnetoresistances.

\section{Method}

What follows is built on these premises: (i) resistivity is a function of magnetic field and temperature T; (ii) scattering processes responsible for GMR are assumed. The first is borne out of experiments and the second ensures implicit inclusion of bulk and interface scatterings, which the various resistivity models account for. In addition, no measurement geometry is preferred since the focus is on the magnitude of GMR.

At a given temperature the standard MR equation:

$$
R(B, T)=1-\frac{\rho^{\uparrow \uparrow}(B, T)}{\rho^{\uparrow \downarrow}\left(B_{o}, T\right)}
$$

where $\rho^{\uparrow \uparrow}(\mathrm{B}, \mathrm{T})$ is parallel alignment resistivity at field $\mathrm{B}$ and temperature $\mathrm{T}$ and $\rho^{\uparrow \downarrow}\left(\mathrm{B}_{\mathrm{o}}, \mathrm{T}\right)$ is antiparallel alignment resistivity at the reference field $B_{0}$ and temperature T. Equation 1 can be presented in the form:

$$
\ln (1-x)=-x-\frac{x^{2}}{2 !}-\frac{x^{3}}{3 !}-\ldots
$$

where $\mathrm{x}=\rho(\mathrm{B}) / \rho_{\mathrm{Bo}}$. The later denotations have been used for parallel and antiparallel resistivity since $\mathrm{B}_{\mathrm{o}}$ and $\mathrm{T}$ are fixed. Equation 2 holds for a number $|\mathrm{x}| \leqslant 1$ and is physically admissible in the present case since GMR lies between 0 and 1 . The expansion may be approximated to a linear function of B since higher powers tend to zero and physically correspond to deviations from Ohm's law. Therefore, it is reasonable to consider the line segment linking two points in $\mathrm{x}$ vs. B plane given by:

$$
d s=\left(1+x^{\prime 2}\right)^{\frac{1}{2}} d B
$$

where $x^{\prime}=\frac{1}{\rho_{B_{0}}} \frac{d \rho(B)}{d B}$.

The corresponding Euler-Lagrange equation gives resistivity:

$$
\rho(B)=\rho_{B_{o}}+\alpha \rho_{B_{o}}\left(B-B_{o}\right)
$$

Here $\alpha$ is a magnetic field coefficient of resistivity. If Mathiessen's rule is assumed valid in a metallic multilayer, contributions by thermal excitations can be added to equation 4 . The contributions include phonon scattering which is proportional to $\mathrm{T}^{3}$ [7], magnon scattering proportional to $\mathrm{T}^{2}$ [8] and Bragg scattering which is proportional to $\mathrm{T}$ and defines "ideal resistivity". However, as temperature is fixed, $\rho_{\mathrm{Bo}}$ at prevailing temperature accommodates their effects.

Now, normalizing equation 4 with zero field resistivity, $\rho_{\mathrm{o}}$, and using $\ln (1-\mathrm{x}) \cong-\mathrm{x}$ (equation 2 ) we have:

$$
R(B)=R_{B_{o}} \exp \left[\alpha \ln R_{B_{o}}\left(B-B_{o}\right)\right]
$$

as the model for variation of GMR with magnetic field. $R_{B o}$ is GMR at a reference field, $\alpha^{\prime}=\alpha \ln R_{B o}$ is magnetic coefficient of GMR. GMR may be reported for positive range of field or in the range of $-\mathrm{B}$ to $\mathrm{B}$, in which case the left region is just an image of the right, hence, characteristics of the structure are completely embodied in the right region, i.e. the positive range. In either case the standard magnetoresistance equation applies and, consequently, equation 5 is valid. The model is a solution to the initial value problem, which is exactly what GMR is: a drastic change from initial resistivity, thus, it has the series form:

$$
R(B)=\sum_{n=0}^{\infty} a_{n}\left(B-B_{o}\right)^{n}
$$

where $a_{n}=(n !)^{-1} d^{n} R(B) / d B^{n}$.

In light of GMR being a function of $\mathrm{B}$, the coefficient shows the ease a multilayer is magnetized. The exponent $\alpha \mathrm{B}$ is analogous to $\chi_{\mathrm{B}}$, i.e. the magnetization of a material, where $\chi$ is susceptibility. The magnetization saturates at the saturation field $\mathrm{B}_{\mathrm{s}}$ at which there is no further redistribution of spin channels, otherwise the magnetization would increase indefinitely with increasing B. It is at this point that GMR attains asymptotic value and parallel alignment resistivity is half the antiparallel alignment value [9]. Thus, $\alpha^{\prime}$ is interpreted as rationalized susceptibility and $\mathrm{B}_{\mathrm{s}}$ is taken to be the rationalizing factor such that: 


$$
\alpha^{\prime}=\frac{\chi_{p}}{B_{s}}
$$

$\chi_{p}$ is the spin paramagnetic susceptibility caused by itinerant electrons. It should be noted that the Landau component of the susceptibility is neglected, as it is much smaller than $\chi_{p}$ for free electrons and inversely proportional to effective electron mass. The spin redistribution follows from [10]:

$$
M=\mu_{B}\left(N^{\uparrow}-N^{\downarrow}\right)=\chi_{p}\left(B-B_{o}\right)
$$

where $\mu_{\mathrm{B}}$ is the Bohr magneton and $\mathrm{N}^{\uparrow, \downarrow}$ the numbers of spin-up and spin-down electrons. Consequently, the effective Fermi vector, $\mathrm{k}_{\mathrm{F}}$, in the multilayer and density of states, $\mathrm{D}\left(\mathrm{E}_{\mathrm{F}}\right)$, at Fermi level are given by [10]:

$$
k_{F}=\frac{4 \pi^{2} \chi_{p} m^{2} c^{2}}{m^{*} e^{2}}
$$

and

$$
D\left(E_{F}\right)=\frac{\chi_{p}}{2 \mu_{B}^{2}}
$$

Here, $\mathrm{m}, \mathrm{m}^{*}$ are electron mass and effective electron mass, $\mathrm{c}$ is the speed of light and e electronic charge. Mathematically, equation 8 warrants continuous increment in magnetization and spin redistribution with increasing magnetic field, however, the physical constraint of saturation field imposes maximum admissible value on them. That is, at saturation field, the magnetization and $\mathrm{N}^{\uparrow}$ $\mathrm{N}^{\downarrow}$ have maximum values. The case of magnetization is obvious from magnetization curve of a multilayer. Therefore, with equation 7 and equation 8, the model can be expressed in terms of magnetization:

$$
R(B)=R_{B_{o}} \exp a M
$$

where $\mathrm{a}=\ln \mathrm{R}_{\mathrm{Bo}} / \mathrm{B}_{\mathrm{s}}$.

\section{Application}

Here, we report the results of the application of equation 5,7 to 10 . The material properties of interest are susceptibility, spin redistribution, effective Fermi vector and density of states of some reported multilayers. Our case studies are $\mathrm{Co} / \mathrm{Cu}[11,13], \quad \mathrm{Co} / \mathrm{Ag} / \mathrm{NiFe} / \mathrm{Ag}$ [12] and $\mathrm{Fe} / \mathrm{Cr}$ [9]. The model equation 5 was implemented in an optimization program involving the Levenberg-Marquardt method that used reported GMR of the structures to deduce the magnetic coefficient $\alpha^{\prime}$. The latter, gave the susceptibility and spin redistribution through equation 7 and equation 8 . The Fermi vectors and density of states were evaluated in free electron approximation in which effective electron mass was taken as equal to free electron mass $\left(9.1 \times 10^{-31} \mathrm{~kg}\right)$ irrespective of electron spin. In case, where GMR was given in the field range of $-\mathrm{B}$ to $\mathrm{B}$, only the right region was considered since the left part was a mirror image and did not affect properties of the system. The results are summarized in Fig. 1 to Fig. 4, Table 1 and Table 2.

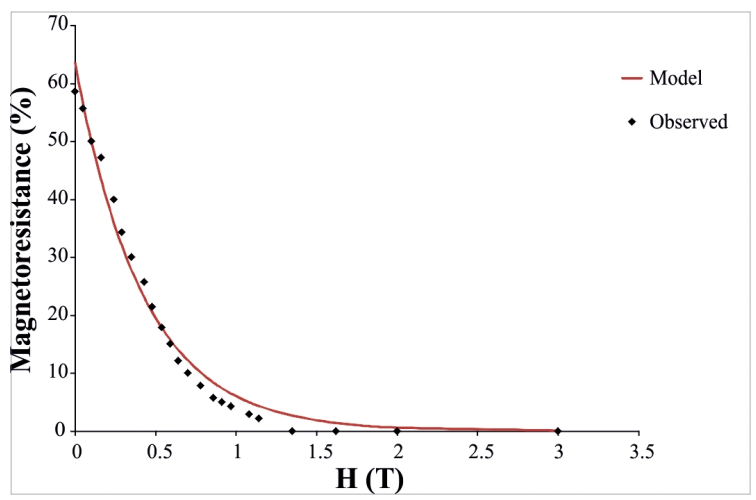

Fig. 1. GMR of $\mathrm{Co}(10 \AA) / \mathrm{Cu}(10 \AA)$ at $4.2 \mathrm{~K}$ and $0 \mathrm{GPa}$. Dotted line is the value reported in the literature [13]. Solid line is obtained from the response model.

The optimization program was implemented on each of the structures to determine their magnetic coefficient $\alpha$. All the fits had coefficient of determination of 0.998 and chi-square well below 0.05 alpha limit. Fig. 1 is an example of such fit with chi-square of 0.012. In every case, instead of fixing $\mathrm{R}_{\mathrm{Bo}}$ to an obvious literature value, it was made adjustable. The deduced values showed minimal deviations from the real ones, for instance $\mathrm{R}_{\mathrm{Bo}}$ for the sample in Fig. 1 had residual of -0.05 . Moreover, there were no significant changes in the fit and $\alpha^{\prime}$ when $\mathrm{R}_{\mathrm{Bo}}$ was fixed to observed values. 
Table 1. Magnetic coefficient $\left(\alpha^{\prime}\right)$ of investigated multilayers.

\begin{tabular}{lll}
\hline Multilayer & ${ }^{*} \alpha\left(\mathrm{T}^{-1}\right)$ & Geometry \\
\hline \hline Electrodeposited [11] & $-1.40\left(\mathrm{t}_{\mathrm{Cu}}=1.0 \mathrm{~nm}\right)$ & CIP \\
$\mathrm{Co}(2 \mathrm{~nm}) /\left.\mathrm{Cu}\left(\mathrm{t}_{\mathrm{Cu}}\right)\right|_{300}$ & $-1.72\left(\mathrm{t}_{\mathrm{Cu}}=3.2 \mathrm{~nm}\right)$ & $\mathrm{CIP}$ \\
\hline $\mathrm{V}$-grooved substrate [12] & -2.95 & $\mathrm{CIP}$ \\
$\mathrm{Co} / \mathrm{Ag} / \mathrm{NiFe} / \mathrm{Ag}$ & -3.03 & $\mathrm{CAP}$ \\
\hline Ion beam sputtering [13] & $-0.72($ at $0 \mathrm{GPa})$ & $\mathrm{CIP}$ \\
$\mathrm{Co}(10 \AA) /\left.\mathrm{Cu}(10 \AA)\right|_{15}$ & $-0.89($ at $2 \mathrm{GPa})$ & $\mathrm{CIP}$ \\
\hline $\mathrm{Molecular}$ beam epitaxy [9] & -0.319 & $\mathrm{CIP}$ \\
$\mathrm{Fe}(30 \AA) /\left.\mathrm{Cr}(9 \AA)\right|_{40}$ & & \\
\hline${ }^{*}$ The negative sign shows that GMR is a decay function of B but not that $\alpha$ is negative which would have led to \\
unacceptable negative parameters in Table 2.
\end{tabular}

Table 2. Susceptibility, Fermi vector and density of state of investigated multilayers.

\begin{tabular}{llll}
\hline Multilayer & $\begin{array}{l}\text { Susceptibility } \\
\chi_{p}\end{array}$ & $\begin{array}{l}\text { Fermi vector } \\
\left(10^{14} \mathrm{~cm}^{-1}\right)\end{array}$ & $\begin{array}{l}\text { Density of states } \\
\left(10^{46} \mathrm{~J}^{-1} \cdot \mathrm{m}^{-3}\right)\end{array}$ \\
\hline \hline Electrodeposited [11] & $1.87\left(\mathrm{t}_{\mathrm{Cu}}=1.0 \mathrm{~nm}\right)$ & 2.62 & 2.18 \\
$\mathrm{Co}(2 \mathrm{~nm}) /\left.\mathrm{Cu}\left(\mathrm{t}_{\mathrm{Cu}}\right)\right|_{300}$ & $2.29\left(\mathrm{t}_{\mathrm{Cu}}=3.2 \mathrm{~nm}\right)$ & 3.21 & 2.66 \\
\hline V-grooved substrate [12] & $1.03(\mathrm{CIP})$ & 1.44 & 1.19 \\
$\mathrm{Co} / \mathrm{Ag} / \mathrm{NiFe} / \mathrm{Ag}$ & $1.06(\mathrm{CAP})$ & 1.49 & 1.23 \\
\hline Ion beam sputtering [13] & $0.69(0 \mathrm{GPa})$ & 0.968 & 0.803 \\
$\mathrm{Co}(10 \AA) /\left.\mathrm{Cu}(10 \AA)\right|_{15}$ & $0.91(2 \mathrm{GPa})$ & 1.28 & 1.05 \\
\hline Molecular beam epitaxy $[9]$ & 0.64 & 0.448 & 0.371
\end{tabular}

$\mathrm{Fe}(30 \AA) /\left.\mathrm{Cr}(9 \AA)\right|_{40}$

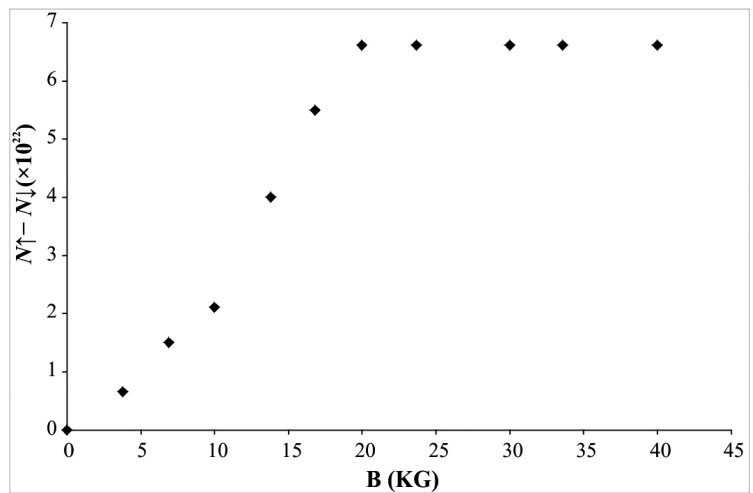

Fig. 2. Redistribution of spin channels in $\mathrm{Fe}(30 \AA) /\left.\operatorname{Cr}(9 \AA)\right|_{40}$ (original multilayer from the literature [9]).

On this account, the model describes very well the variation of GMR with magnetic field. Equation 11 presents an alternative approach with similar results since $\alpha \mathrm{M}$ reduces to the argument of the

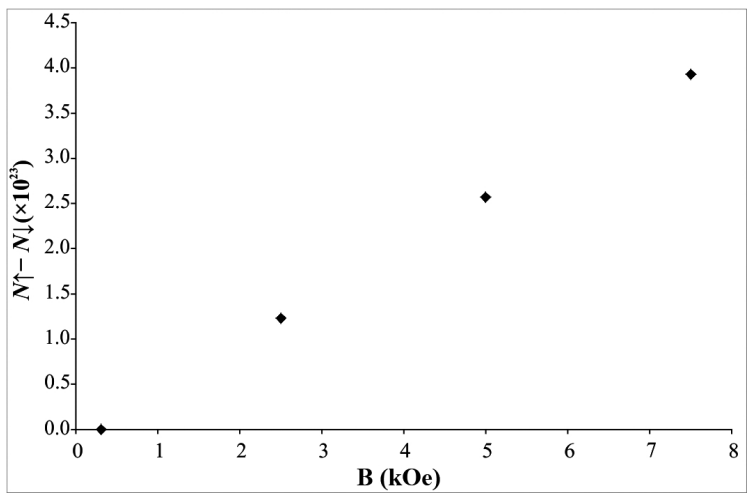

Fig. 3. Redistribution of spin channels in $\mathrm{Co}(2 \mathrm{~nm}) /\left.\mathrm{Cu}(3.2 \mathrm{~nm})\right|_{300}$ (original multilayer from the literature [11]).

exponential term in equation 5 through equation 7 , 8 and $\alpha=\ln R_{\mathrm{Bo}} / \mathrm{B}_{\mathrm{s}}$. Fig. 2 to Fig. 4 are the spin redistributions in $\mathrm{Fe} / \mathrm{Cr}$ and $\mathrm{Co} / \mathrm{Cu}$ that were evaluated with the determined $\alpha^{\prime}$ and equation 7 and 


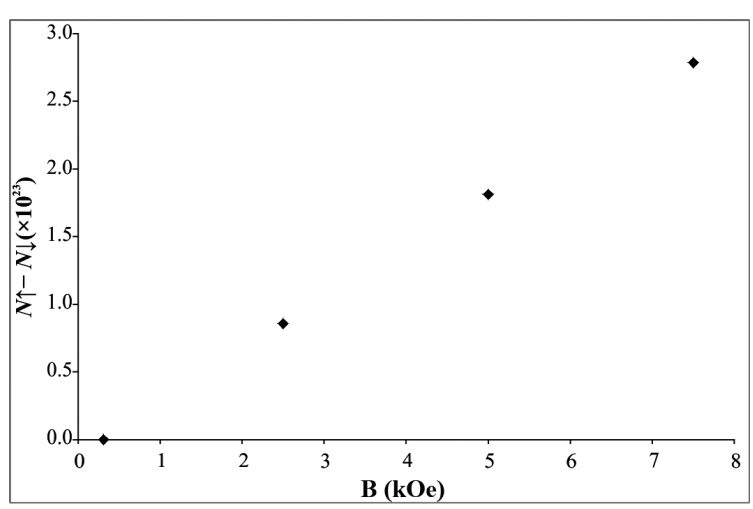

Fig. 4. Redistribution of spin channels in $\mathrm{Co}(2 \mathrm{~nm}) /\left.\mathrm{Cu}(1 \mathrm{~nm})\right|_{300}$ (original multilayer from the literature [11]).

equation 8 . Mathematically, equation 8 suggests a continuing increase of spin redistribution and magnetization as long as $\mathrm{N}^{\uparrow}>\mathrm{N}^{\downarrow}$ (which holds true for metals), if we neglect the observable fact that magnetization always saturates. This physical constraint was imposed on the referenced equation for the systems in Fig. 2 to Fig. 4 and the mathematical character of equation 8 was well defined at low fields. In Fig. 2, the spin redistribution levels off at $20 \mathrm{KG}$, where Fe30 $\AA /\left.\mathrm{Cr} 9 \AA\right|_{40}$ attains maximum GMR [9]. Using Stoner criterion, a probable reason for this is that the magnitude of the shift in energy $\left|\mu_{\mathrm{B}} \mathrm{B}\right|$ cannot exceed the zero field energy, otherwise it would result in a limitless shift in energy of the conduction electrons with the implication that electrons may escape the structure at some large field. In other words, up-spin energy $\mathrm{E}^{\uparrow}=\mathrm{E}(\mathrm{k})-$ $\mu_{\mathrm{B}} \mathrm{B}$ cannot be less than zero and down-spin energy $E^{\downarrow}=E(k)+\mu_{B} B$ cannot exceed twice the zero field energy, $\mathrm{E}(\mathrm{k})$. Then, at saturation field, $\mu_{\mathrm{B}} \mathrm{B}=\mathrm{E}(\mathrm{k})$ which implies that zero field energy determines saturation field. At any instance of applied field, the sum of energies of up- and downspin electrons is twice $\mathrm{E}(\mathrm{k})$. This is a consequence of Pauli exclusion principle and Fermi vector being intrinsically, the average of up- and down-spin Fermi vectors [15]. The redistribution of spins in Fig. 3 and Fig. 4 increases, as expected, with magnetization up to $7.5 \mathrm{kOe}$ at which the respective GMR tends towards its asymptotic value [11]. This field was used in determining the susceptibilities of the concerned structures. These figures demonstrate that not only is spin redistribution present in GMR but not all antiparallel spins are aligned in direction of applied field even at saturation field. Why this happens is not yet clear.

Magnetic coefficients and susceptibilities in Table 1 and Table 2 are alternative indicators of how quickly a material is magnetized. The attention was focused on the susceptibility because of the role of itinerant electrons in GMR. Table 2 shows clearly that susceptibility is dependent on thickness and pressure. Now, a major property of metallic structures is the antiferromagnetic exchange coupling (AF). This coupling affects the saturation field and decays with increasing thickness of nonmagnetic layer in metallic superlattices. Thus, it can be seen that in $\mathrm{Co}(2 \mathrm{~nm}) /\left.\mathrm{Cu}(\mathrm{t})\right|_{300}$, where the coupling is reduced for $\mathrm{t}_{\mathrm{Cu}}=3.2 \mathrm{~nm}$, the susceptibility is higher. Interestingly, in $\operatorname{Co}(10 \AA) /\left.\mathrm{Cu}(10 \AA)\right|_{15}$ with the $\mathrm{Cu}$ thickness equivalent to $1 \mathrm{~nm}$ as in $\mathrm{Co}(2 \mathrm{~nm}) /\left.\mathrm{Cu}(\mathrm{t})\right|_{300}$, where AF coupling is expectedly high, the susceptibility is anticipated to be invariant with pressure. However, the noticeable difference between its value at $2 \mathrm{GPa}$ and $0 \mathrm{GPa}$ (ambient pressure) suggests that external pressure can suppress AF coupling. Since nonmagnetic layer modulates AF coupling, using the one-band model [16] in which the coupling is dependent on nonmagnetic layer thickness, the effect of pressure is attributed to shrinking of the thickness with increasing pressure. However, suppression of AF coupling may not necessarily lead to enhanced GMR at elevated pressure because of differing effects that can result from modification of interface roughness. An increase in roughness enhanced GMR in DC-magnetron sputtered $\mathrm{Fe} / \mathrm{Cr}$ [17] but suppressed GMR in molecular beam epitaxy $\mathrm{Fe} / \mathrm{Cr}$ were observed [18]. The involved structure in the present case has lower GMR $(50.5 \%)$ at 2 GPa than at ambient pressure (54.0\%) [13], showing that the principle mechanism is interface scattering. Therefore, though elevated pressure reduced AF coupling leading to higher susceptibility, the attendant flattening of interface roughness caused lowering of GMR. It should be emphasized that a different behavior may be obtained in a structure, where interface roughness 
suppresses GMR. Quantitative description of the AF coupling-pressure relation requires detailed experimental measurements and modification of the one-band model [16] in terms of pressure, especially the aspect of dependence of the thermodynamic potential of holes on the layer thickness. Moreover, in case of $\mathrm{Co} / \mathrm{Ag} / \mathrm{NiFe} / \mathrm{Ag}$, where no changes in external conditions occur (pressure, thickness), the susceptibility is essentially invariant in the geometry of measurement. When this is compared with $\operatorname{Co}(2 \mathrm{~nm}) /\left.\mathrm{Cu}(\mathrm{t})\right|_{300}$ and $\mathrm{Co}(10 \AA) /\left.\mathrm{Cu}(10 \AA)\right|_{15}$ without accounting for AF coupling, it can be seen that the magnitude of susceptibility is dependent on the quality of interface, because the external conditions alter the roughness. Thus, besides its association with AF coupling, susceptibility is a qualitative measure of roughness. In the $\mathrm{Co} / \mathrm{Cu}$ structures studied here, high values correspond to smoother interfaces and lower magnetoresistance in ion beam sputtered $\mathrm{Co} / \mathrm{Cu}$ but higher magnetoresistance in electrodeposited $\mathrm{Co} / \mathrm{Cu}$, hence, interface roughness scattering suppresses GMR in the former but enhances it in the latter. In general, the susceptibility is dependent on AF coupling and interface roughness.

\section{Conclusion}

The model gives a good interpretation of magnetoresistance variation with magnetic field and allows deducing material properties from observed values. For theoretical study, it can be used in conjunction with any resistivity model to arrive at the material properties presented herein. However, the obtained results may differ, especially the Fermi vector, because of the choice of effective electron mass. To the best of our knowledge at time of this work, there have been no reported measured values of the parameters collected in Table 2. The values are open to experimental investigations and may differ since our evaluations used the free electron approximation.

\section{References}

[1] Camley R.E., Barnas J., Phys. Rev. Lett., 63 (1989), 664.

[2] Valet T., Fert A., Phys. Rev. B, 48 (1993), 7099.

[3] Barnas J., Fuss A., Camley R.E., Grunberg P., ZINN W., Phys. Rev. B, 42 (1990), 8110.

[4] Zhang S., LeVy P.M., J. Appl. Phys., 69 (1991), 4786.

[5] Camblong H.E., Phys. Rev. B, 51 (1995), 1855.

[6] Barnas J., Bruynseraede Y., Phy. Rev. B, 53 (1996), 5449.

[7] Wilson A.H., P. Roy. Soc. A-Math. Phy., 167 (1938), 580.

[8] Goodings D.A., Phys. Rev., 132 (1963), 542.

[9] Baibich M.N., Broto J.M., Fert A., DaU VAN N.F., Petroff F., Eitenne P., Creuzet G., Friederich A., Chazelas J., Phys. Rev. Lett., 61 (1988), 2472.

[10] Animalu A.O.E., Intermediate Quantum Theory of Crystalline Solids, Prentice-Hall, New Jersey, 1977.

[11] Kashiwabara S., Jyoko Y., Hayashi Y., Physica $B, 239$ (1997), 47.

[12] Ono T., Shigeto K., Shinjo T., Physica B, 239 (1997), 41.

[13] OOMi G., Sakai T., Uwatoko Y., Takanashi K., Fujimori H., Physica B, 239 (1997), 19.

[14] Binasch G., Grunberg P., Saurenbach F., Zinn W., Phys. Rev. B, 39 (1989), 4828.

[15] Uba J.I., Ekpunobi A.J., Ekwo P.I., Afr. Rev. Phys., 9 (0043) (2014), 339.

[16] Edwards D.M., Mathon J., Muniz R.B., Phan M.S., Phys. Rev. Lett., 67 (1991), 493.

[17] Fullerton E.E., Kelly D.M., Guimpel J., SCHUller I.K., Phys. Rev. Lett., 68 (1992), 859.

[18] Belien P., Schad R., Potter C.D., Verbanck G., Moshchalkov V.V., Bruynseraede Y., Phys. Rev. B, 50 (1994), 9957. 